\title{
Adaptive Medium Access Control for VoIP Services in IEEE 802.11 WLANs
}

\author{
Mamun I. Abu-Tair ${ }^{1}$, Geyong Min $^{1 *}$, Qiang $\mathrm{Ni}^{2}$, Hong Liu ${ }^{3}$ \\ ${ }^{1}$ Department of Computing, School of Informatics, University of Bradford, Bradford, U.K. \\ 2 Electronic \& Computer Engineering Division, School of Engineering and Design, Brunel University, West London, U.K. \\ ${ }^{3}$ Wuhan National Laboratory for Optoelectronics, Huazhong University of Science and Technology, China. \\ Email: \{m.i.a.abu-tair,g.min\}@brad.ac.uk, qiang.ni@brunel.ac.uk,hongliu@hust.edu.cn
}

\begin{abstract}
Voice over Internet Protocol (VoIP) is an important service with strict Quality-of-Service (QoS) requirements in Wireless Local Area Networks (WLANs). The popular Distributed Coordination Function (DCF) of IEEE 802.11 Medium Access Control (MAC) protocol adopts a Binary Exponential Back-off (BEB) procedure to reduce the packet collision probability in WLANs. In DCF, the size of contention window is doubled upon a collision regardless of the network loads. This paper presents an adaptive MAC scheme to improve the QoS of VoIP in WLANs. This scheme applies a threshold of the collision rate to switch between two different functions for increasing the size of contention window based on the status of network loads. The performance of this scheme is investigated and compared to the original DCF using the network simulator NS-2. The performance results reveal that the adaptive scheme is able to achieve the higher throughput and medium utilization as well as lower access delay and packet loss probability than the original DCF.
\end{abstract}

\section{INTRODUCTION}

The rapid increase in the number of Personal Digital Assistants PDA devices, palmtops and compact laptops has made wireless networks popular in practice. Such networks provide a flexible data communication system that can either replace or extend a wired LAN to provide location independent network access between computation and communication devices using waves rather than a cable infrastructure [6]. Wireless networks are becoming more widely recognized as a general-purpose connectivity alternative for a wide range of business organizations owing to its simplicity, scalability, relative ease of integrating wireless access and ability for wireless stations to roam throughout the business organizations with the remaining connected to other existing network resources such as servers, printers, and Internet connections. One of the major types of wireless networks is the infrastructure Wireless Local Area Networks (WLANs), which are distinguished by the use of an access point (AP) [6]. All communication in the infrastructure WLANs come through the AP.

In order to allow the stations to share the wireless medium efficiently, many practical WLANs have widely been deployed following the Institute of Electrical and Electronics Engineers (IEEE) 802.11 standards ratified in 1997 that operate at data rates up to $2 \mathrm{Mbps}$ in the $2.4-\mathrm{GHz}$ Industrial, Scientific and Medical (ISM) band. But the most general

\footnotetext{
* Corresponding author. The research was supported in part by the

UK EPSRC Grant EP/C525027/1.
}

business requirements cannot be well supported by the slow data rate of the original IEEE 802.11 standard. Recognizing the critical need to support higher data-transmission rates, the IEEE ratified both $802.11 \mathrm{a}$ and $802.11 \mathrm{~b}$ standards with the rates up to 54 and $11 \mathrm{Mbps}$ in the 5 and 2.4-GHz ISM band, respectively $[6,18]$. Moreover, both standards specify the operation of the Medium Access Control (MAC) protocol, which is responsible for controlled access to the transmission medium. The most important purpose of this protocol is to enable the capacity of transmission media to be utilized in an efficient manner by wireless network devices.

The IEEE 802.11 MAC protocol offers two different methods to support shared access to wireless channels; a Distributed Coordination Function (DCF) and an optional Point Coordination Function (PCF) [6]. At the present time, the DCF is the dominant MAC mechanism implemented in the IEEE 802.11-compliant products. The DCF is based on the Carrier Sense Multiple Accesses (CSMA) mechanism, which is a contention-based protocol making certain that the stations first sense the medium prior to data-transmission. Moreover, the DCF applies a collision avoidance (CA) mechanism which can reduce the collision probability using an additional random binary exponential time called back-off time. The main objective of CSMA/CA is to avoid stations transmitting at the same time, which can lead to collisions and corresponding retransmissions $[6,8,9]$. In addition to the common CSMA/CA techniques, the DCF further reduces the possibility of collisions and improves data delivery reliability by adding acknowledgement frames and optional channel reservation frames (i.e., Request-To-Send and Clear-To-Send) to the exchange sequences of its data frames. Different from DCF, the optional coordination function PCF is a centralized scheme designed for infrastructure networks that have a point coordinator operating at the Access Point (AP) to poll and select the next wireless station for data-transmission [6].

The enhancement of the IEEE 802.11 MAC protocol has attracted numerous research efforts $[3,7,16]$. Wu et al. [16] have proposed a mechanism to enhance the throughput of DCF by adjusting the scheme of resetting the contention window. Lin and Pan [7] have introduced a Tender back-off algorithm, which adds two more back-off stages to the original BEB scheme. They have shown that this mechanism can improve the throughput of DCF. However, the authors did not 
study other QoS performance measures of the proposed mechanism, such as packet delay and loss probability. Chatzimisions et al. [3] have proposed a Double Increment Double Decrement (DIDD) back-off algorithm which decreases the size of contention window half after a successful transmission rather than resetting it to the minimum value. They have conducted an extensive performance study to demonstrate the efficiency of this algorithm.

In order to enhance the performance of the DCF protocol, we propose a new adaptive MAC scheme, which takes the traffic loads of the stations into account. This scheme uses the recent collision rate as a threshold to switch between two different increasing functions (i.e., exponential and quadratic) in the case of collision. The exponential increasing function is adopted when the mobile station works under light traffic loads while the quadratic increasing function is used when the wireless station has heavy loads. The performance results based on simulation experiments demonstrate that the adaptive MAC scheme outperforms the original DCF in terms of throughput, access delay, medium utilization and packet loss probability.

The rest of this paper is organized as follows: Section II introduces the DCF MAC protocol of the IEEE 802.11 wireless networks and then presents the idea and algorithm of the new adaptive MAC scheme. Section III describes the experimental scenarios and the setting of simulation parameters. Section IV presents and analyses the performance results obtained from simulation experiments. Finally, Section V concludes this study.

\section{BACKGROUND}

\section{A. Distributed Coordination Function (DCF)}

The contention-based DCF is the basic MAC mechanism of IEEE 802.11 WLANs. Similar to other contention-based MAC protocols, DCF relies on a Carrier Sense Multiple collision Access with Collision Avoidance (CSMA/CA) algorithm to access the shared medium. A station having packets ready for transmission senses whether or not the medium is busy. If it has been idle for longer than the minimum duration called DCF Interference Space (DIFS), the station starts transmission immediately. Otherwise, a back-off time is chosen randomly from the interval $[0, c w]$, where $c w$ represents the contention window $[8,9]$. The stations start down-counting its back-off counter by one only if the medium has been detected idle for at least a DIFS. If the medium gets busy due to other transmissions, the back-off counter pauses down-counting and resumes when the medium has been sensed idle for DIFS again $[6,8]$. Transmission may proceed when back-off counter has reached zero. Upon detection of a collision, i.e., when the back-off counter of two or more stations reaches zero at the same time, the contention window is doubled according to $c w_{i}=2^{k+i-1}-1$ where $i$ is the number of attempts to transmit the frame and $k$ is a constant defining the minimum contention window $c w_{\min }=2^{k}-1$ [8]. When the destination station receives frame successfully, it sends an acknowledgment (ACK) frame back to the source station after a Short Inter frame Space (SIFS) duration. Additionally, to alleviate the hidden station problem, DCF uses optional Request-to-Send/Clear-to-Send (RTS/CTS) frames before packet transmission [6]. This process is illustrated in Figure 1.

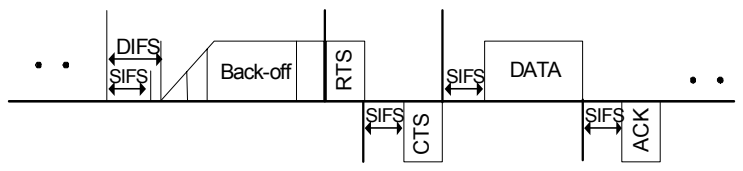

Figure 1. RTS/CTS of IEEE 802.11 DCF.

\section{B. An adaptive MAC scheme}

IEEE 802.11 DCF adopts an exponentially back-off algorithm to handle the transmission collision between different wireless stations. However, the DCF does not consider the station situation whether it is under heavy traffic loads or not. In the case of few contending stations, the DCF tends to work well in an efficient manner. However, when the number of stations increases, the rapid decrease of contention window in the case of successful transmission and the slow increase under transmission collision can lead to significant performance degradation. To handle this problem, this paper proposes an adaptive MAC scheme to control the wireless medium, which uses the recent collision rate as a threshold to switch between two different increasing functions (i.e., exponential and quadratic) in the case of transmission collision. More specifically, this scheme operates exactly as DCF with the exponential increasing function when the wireless station works under the light traffic loads (i.e. the collision rate is low). However, it uses the quadratic increasing function when the wireless station has the heavy traffic loads and the collision rate is high. This scheme can avoid the increasing number of collisions caused by the unsuitable small contention window sizes.

Similar to $[5,10,13,17]$, the recent collision rate can be calculated by dividing the time domain of the network connection into continuous intervals with the specific number of slot times. At the end of each interval, the mobile station computes the collision rate as

$$
\beta_{\text {curr }}^{i}=\frac{(\text { num_collisions })^{i}}{(\text { num_packets_sent })^{i}}
$$

where (num_collisions $)^{i}$ and $(\text { num_packets_sent })^{i}$ are the number of collisions and the number of packets successfully sent at the $i^{\text {th }}$ interval, respectively.

Furthermore, in order to precisely represent the long-term and short-term network conditions, an Exponentially Weighted Moving Average (EWMA) method is used as a smoother for the estimated value of the collision rate. In particular, let $\beta_{\text {ave }}^{i}$ denote the average collision rate after $i^{\text {th }}$ interval period. $\beta_{\text {ave }}^{i}$ is given by

$$
\beta_{\text {ave }}^{i}=(1-\gamma)^{*} \beta_{\text {curr }}^{i}+\gamma^{*} \beta_{\text {ave }}^{i-1} \text { where } 0<\gamma<1
$$


Figure 2 describes the dynamic back-off algorithm used in the adaptive MAC scheme.

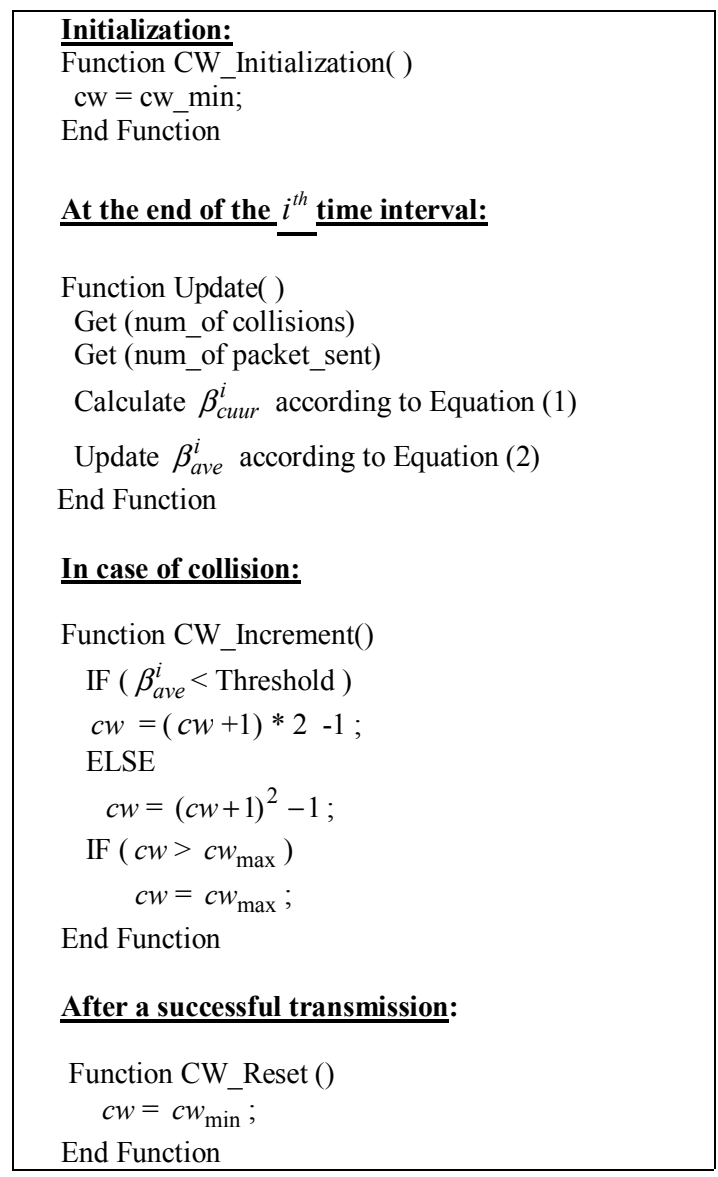

Figure 2. An adaptive MAC scheme back-off algorithm.

\section{SimUlation SCENARIO AND CONFIGURATION}

The well-known network simulator NS-2 [11] has been adopted to conduct our simulation experiments. This section presents the experimental scenario and discusses how to configure simulation parameters. The simulation scenario studied in this research was designed to investigate the performance of the adaptive MAC scheme in hot spot areas of WLANs.

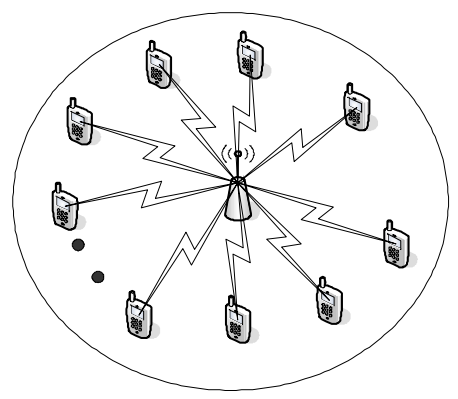

Figure 3. AP with mobile stations.
The scenario is composed of up to 75 wireless stations. Each wireless station operates under the IEEE 802.11a standard at a data rate of $24 \mathrm{Mbps}$ [8]; the setting of physical layer (PHY) and MAC parameter is listed in Table I. All stations are located within a Basic Service Set (BSS) such that every station is able to detect a transmission from others. Each station generates packets according to the bursty ON/OFF process in order to capture the characteristics of the voice applications. In general, the talkspurt and silence durations of voice ON/OFF process follow an exponential distribution with the mean of $1.004 \mathrm{~s}$ and 1.587s [4], respectively. During the talkspurts period, each source generates the packets with the size of 280 bytes, corresponding to a constant sending rate of $64 \mathrm{Kbit} / \mathrm{s}$. Given that each station may involve multiple voice sources, we consider that there are three voice sources at each station.

TABLE I. Ns-2 Phy PARAmeters for IEeE 802.11a.

\begin{tabular}{|l|l|}
\hline SlotTime & $9 \mu \mathrm{s}$ \\
\hline CCATime & $3 \mu \mathrm{s}$ \\
\hline RxTxTurnaroundTime & $2 \mu \mathrm{s}$ \\
\hline SIFSTime & $16 \mu \mathrm{s}$ \\
\hline PreambleLength & 96 bits \\
\hline PLCPHeaderLength & 40 bits \\
\hline PLCPDataRate & $6 \mathrm{Mbps}$ \\
\hline$c w_{\text {min }}$ & 15 \\
\hline$c w_{\text {max }}$ & 1023 \\
\hline$\gamma$ & 0.8 \\
\hline Collision Threshold & 0.5 \\
\hline
\end{tabular}

\section{Performance Analysis And Evaluation}

The simulation experiments aim to investigate the performance of the adaptive MAC scheme in the IEEE 802.11 WLANs in terms of throughput, access delay, medium utilization and packet loss probability in the presence of voice traffic. The average throughput is calculated as the amount of data actually delivered to the destination during each time unit. Many factors affect the throughput, including the efficiency of collision avoidance, medium utilization, latency, and control overhead. The access delay is defined as the time elapsed from the arrival of a packet from the higher layer to the MAC layer until the start of the successful transmission on wireless medium. We measure the access delay to find out how well the adaptive MAC scheme accommodates the VoIP services. Medium utilization is referred to the percentage of time that is used for successful transmission. Moreover, the packet loss probability is calculated as the ratio of the number of lost packets over the number of generated packets.

\section{A. Throughput}

As an effort to investigate the performance of the adaptive MAC scheme, Figure 4 compares the throughput of the original DCF and the adaptive MAC scheme versus the number of wireless stations. As shown in the figure, when the system is under light traffic loads, the throughput of the 


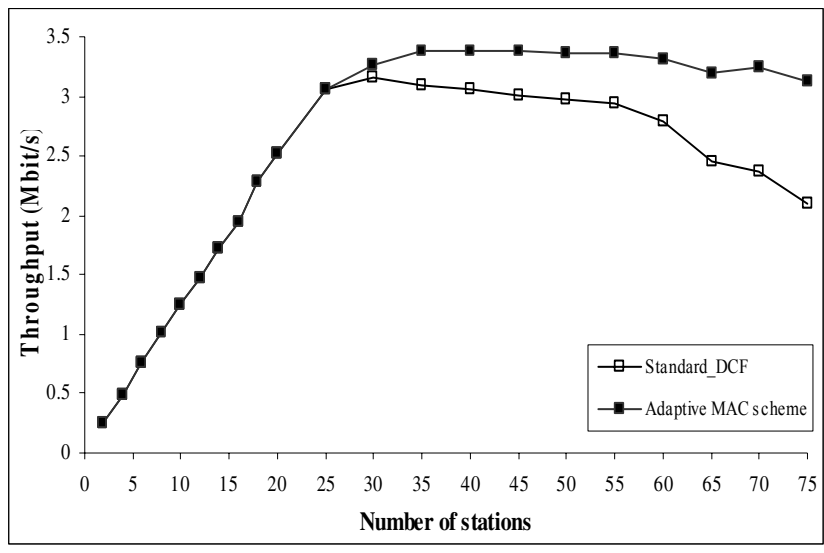

Figure 4. Throughput comparison between the original DCF and adaptive MAC scheme.

original DCF is close to that of the adaptive MAC scheme. This is due to the fact that the adaptive MAC scheme operates similarly to the original DCF under light traffic loads. However when the number of stations exceeds 25 active stations, the throughput of the DCF begins to decrease while the throughput of the adaptive MAC scheme keeps increasing until there are 35 active stations. Moreover, Figure 4 shows the throughput of the adaptive scheme is higher than the original DCF under heavy traffic loads (when there are more than 25 wireless stations). For example, when there are 75 active stations the throughput is around $3.25 \mathrm{Mbit} / \mathrm{s}$ when the adaptive scheme is used and around $2.1 \mathrm{Mbit} / \mathrm{s}$ when the original DCF is used, respectively. It is worth noting that the setting of a small size of contention window and slow increasing of contention window in the case of collision could degrade the system throughput when there are a large number of contenting stations. As a result, the adaptive MAC scheme can perform similarly to the original DCF under the light traffic loads and outperform under the heavy loads in terms of throughput.

\section{B. Access Delay}

Figure 5 presents a comparison of the mean access delay between the original DCF and adaptive MAC scheme. As shown in the figure, the mean access delay of the adaptive MAC scheme is comparable to that of the original DCF under light traffic loads and becomes lower under heavy loads. This is due to the use of the quadratic increasing functions in the case of collision in the adaptive MAC scheme, which provides the contenting station with the large contention windows. This mechanism can overcome the increasing delay caused by the small contention window, which increases the collision probability. In general, the adaptive scheme maintains around $10 \%$ lower of the mean access delay than the original DCF for most cases under the heavy traffic loads. Moreover, using two different increasing functions by the adaptive scheme can provide the wireless stations with a proper contention window size according to the network status (a large contention window when there are more contenting stations and a small one when there are few contenting stations). The use of this

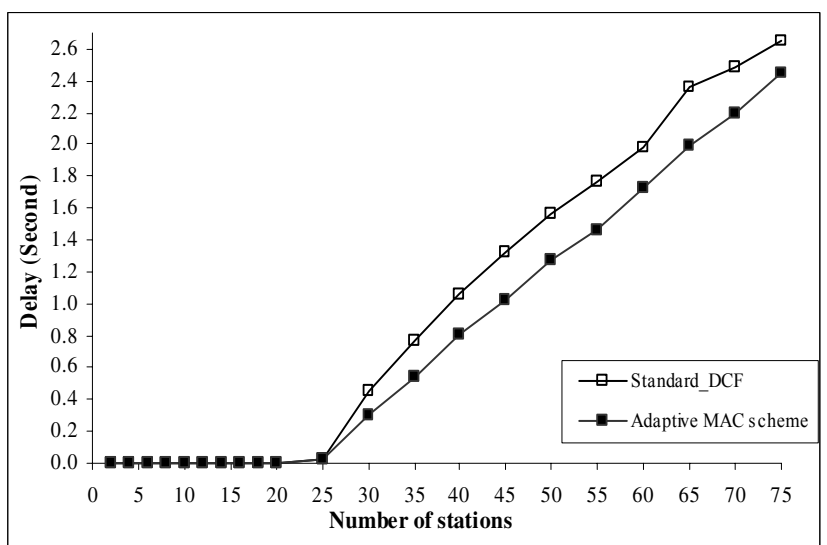

Figure 5. Access delay comparison between the original DCF and adaptive MAC scheme.

technique directly affects the mean access delay. As a result, the adaptive MAC scheme has comparable access delay under the light traffic loads and lower access delay under the heavy loads compared to the original DCF.

\section{Medium Utilization}

In addition to throughput and mean access delay, Figure 6 compares the medium utilization of the original DCF and adaptive MAC scheme. It can be seen that the medium utilization of the original and adaptive MAC scheme keeps increasing as the number of active stations increases until there are 25 and 35 active stations, respectively. After these points, they cannot obtain the increasing medium utilization due to the increasing number of collisions caused by the increase in traffic loads. Additionally, Figure 6 shows that the adaptive MAC scheme maintains the medium utilization higher than the original DCF under heavy traffic loads. This is due to the fewer number of collisions when the adaptive MAC scheme is used than that for the original DCF. For example, the medium utilization of the adaptive MAC scheme is around $38 \%$ while it is around $29 \%$ for the original DCF, when there are 75 stations. It is worth noting that the maximum medium

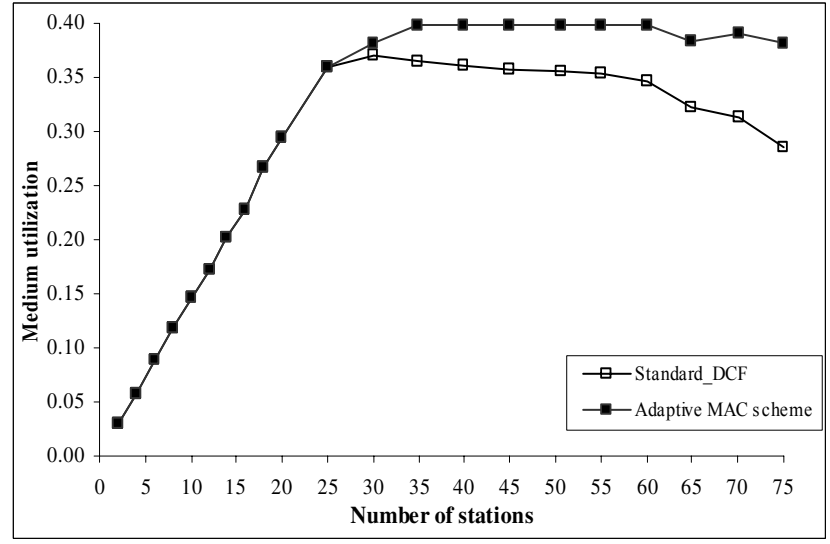

Figure 6. Medium utilization comparison between the original DCF and adaptive MAC scheme. 
utilization of the WLAN is very low due to the large number of contending stations.

\section{Packet Loss Probability}

Figure 7 depicts the packet loss probability of the original DCF and adaptive MAC scheme. From this figure, we can observe that no packet is lost until there are more than 20 active stations. Beyond this point, the packet loss probability of the original DCF and adaptive MAC scheme increases. This is because the queue of both schemes becomes full due to the increasing waiting time for the packets to be transmitted successfully. Moreover, the adaptive scheme has a lower packet loss probability (around 0.6) than that of the original DCF (around 0.78) when there are 70 contending stations. This is because the adaptive scheme can send more packets than the original DCF under heavy traffic loads. Furthermore, both schemes suffer from the high packet loss probability when the traffic load is high. This phenomenon is due to the increasing number of collisions and the limited capacity of the transmission queues, which cannot accommodate more packets. As a result, the adaptive MAC scheme performs better than the original DCF in terms of packet loss probability.

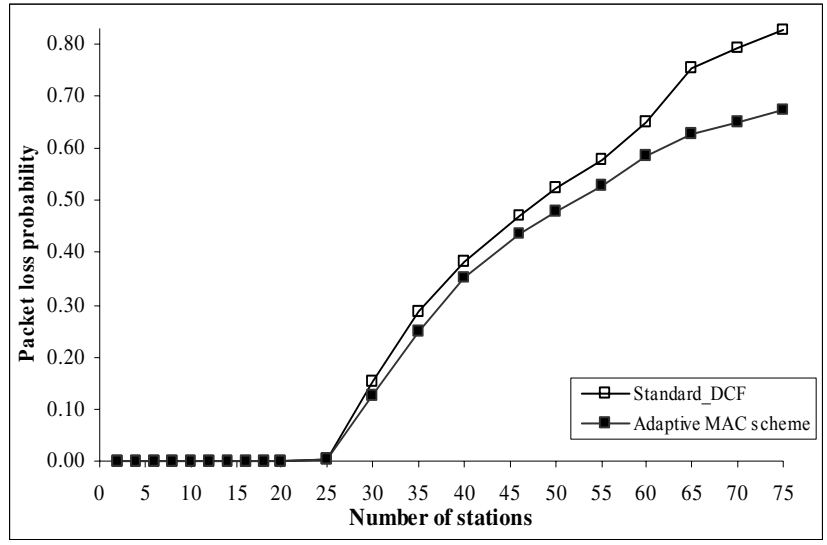

Figure 7. Packet loss probability comparison between the original DCF and adaptive MAC scheme.

\section{CONCLUSIONS}

Wireless Local Area Networks (WLANs) offer the flexible data communication systems to provide location independent network access between computation and communication devices using waves rather than a cable infrastructure. Many MAC protocols have been reported to mange and control the shared wireless medium. This paper has proposed an adaptive MAC scheme in order to enhance the performance of the IEEE 802.11 WLANs. This scheme employs the collision rate as a threshold to switch between an exponential and quadratic increasing function in the case of collision to increase the contention window. The exponential increasing function is used when the network works under the light traffic loads while the quadratic function is used when the traffic load is high and the collision rate is large. Performance evaluation of this adaptive scheme has been conducted using the network simulator NS-2. The performance results have shown that the adaptive scheme outperforms the original DCF in terms of throughput, mean access delay, medium utilization and packet loss probability. More specifically, under the high traffic loads, the throughput of the adaptive scheme increases up to $28 \%$, the medium utilization increases $28 \%$, the access delay decreases $10 \%$ and the packet loss probability decreases $18 \%$.

\section{REFERENCES}

[1] I. Aad and C. Castelluccia, Differentiation mechanisms for IEEE 802.11, Proc.20th Annual Joint Conference of the IEEE Computer and Communications Societies, IEEE (INFOCOM'2001), vol. 1, pp. 209-218, 2001.

[2] J. Beran, R. Sherman, M.S. Taqqu and W. Willinger, Long-range dependence in variable-bit-rate video traffic, IEEE Trans. Communications, vol. 43, pp. 1566-1579, 1995.

[3] P. Chatzimisions, V. Vitasas, A. Boucouvalas and M. Tsoulfa, Achieving performance enhancement in IEEE 802.11 WLANs by using DIDD backoff mechanism, International Journal of Communication Systems, vol. 20, no. 1, pp. 23-41, 2007.

[4] C. Chuah and R. Katz, Characterizing packet audio streams from Internet multimedia applications, Proc. IEEE International Conference on Communication (ICC'2002), vol.2, pp. 1199-1203, 2002.

[5] L. Gannoune, A non-linear dynamic tuning of the minimum contention window (CWmin) for enhanced service differentiation in IEEE 802.11 ad-hoc networks, Proc. Vehicular Technology Conference,(VTC 2006-Spring), vol.3, pp.1266-1271, 2006.

[6] M. Gast, 802.11 wireless networks: the definitive guide, vol.1, O'Reilly, 2002.

[7] I.-H. Lin and J.-Y. Pan, Throughput analysis of a novel backoff algorithm for IEEE 802.11 WLANs, Proc. Wireless Telecommunications Symposium, pp. 85- 90, 2005.

[8] S. Mangold, S. Choi, G. Hiertz, O. Klein and B. Walke, Analysis of IEEE 802.11e for QoS support in wireless LANs, IEEE Wireless Communications, vol. 10, no. 6, pp. 40-50, 2003.

[9] S. Mangold, S. Choi, Peter May, O. Klein, G. Hiertz and L. Stibor, IEEE $802.11 \mathrm{e}$ wireless LAN for quality of service, Proc. European Wireless Conference, Italy, 2002.

[10] Q. Ni, Performance analysis and enhancements for IEEE 802.11e wireless networks, IEEE Network, vol. 19, no. 4, pp. 21-27, 2005.

[11] Ns-2 simulator, available on: www.isi.edu/nsnam

[12] V. Paxson and S. Floyd, Wide-area traffic: the failure of Poisson modeling, IEEE/ACM transaction on networking, vol. 3, no. 3, pp. 226-244, 1995.

[13] L. Romdhani, Q. Ni and T. Turletti, Adaptive EDCF: enhanced service differentiation for IEEE 802.11 wireless ad hoc networks, Proc. IEEE Wireless Communications and Networking Conference (WCNC 2003), New Orleans, Louisiana,USA, 2003.

[14] Z. Tao and S. Panwar, Throughput and delay analysis for the IEEE $802.11 \mathrm{e}$ enhanced distributed channel access, IEEE Trans. Communications, vol. 54, no. 4, pp. 596-603, 2006.

[15] O. Tickoo and B. Sikdar, On the impact of IEEE 802.11 MAC on traffic characteristics, IEEE Journal on Selected Areas in Communications, vol. 21, no. 2, pp. 189-203, 2003.

[16] H. Wu, S. Cheng, Y. Peng, K. Long and J. Ma, IEEE 802.11 distributed coordination function (DCF): analysis and enhancement, Proc. IEEE International Conference (ICC), vol.1, pp. 605-609, 2002.

[17] H. Zhu, G. Cao, A. Yener and A. Mathias, EDCF-DM: a novel enhanced distributed coordination function for wireless ad hoc networks, Proc. IEEE International Conference on Communication (ICC'2004), vol.7, pp. 3886-3890, 2004

[18] IEEE WG 802.11, Part 11: Wireless LAN Medium Access Control (MAC) and Physical Layer (PHY) Specifications, ISO/IEC 880211:1999(E), IEEE Standards 802.11, 1999. 